\title{
ANTIOXIDATIVE SYSTEM OF BOVINE FOLLICLES REGARDING STAGE OF ESTROUS CYCLE AND FOLLICULOGENESIS
}

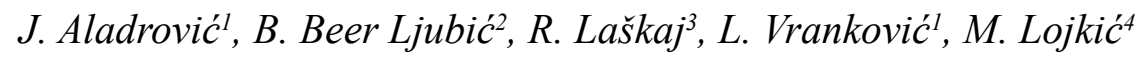 \\ jasna.aladrovic@vef.hr
}

${ }^{1}$ University of Zagreb, Faculty of Veterinary Medicine,

Department of Physiology and Radiobiology,

Heinzelova ul., 55, Zagreb, 10000, Croatia

${ }^{2}$ University of Zagreb, Faculty of Veterinary Medicine, Internal Diseases Clinic,

Heinzelova ul., 55, Zagreb, 10000, Croatia

${ }^{3}$ University Hospital for Infectious Diseases "Dr Fran Mihaljevic",

Mirogojska cesta 8, Zagreb, 10000, Croatia

${ }^{4}$ University of Zagreb, Faculty of Veterinary Medicine, Reproduction and Obstetrics Clinic,

Heinzelova ul., 55, Zagreb, 10000, Croatia

Oxidative stress is important for promoting the oocyte maturation and ovulation within the follicle. The aim of the study was to examine activities of the superoxide dismutase (SOD), glutathione peroxidase (GPX) and biological antioxidant potential (BAP) involved in protection against free oxygen radicals and concentration of reactive oxygen metabolites (ROMs) in bovine follicular fluid.

Bovine ovaries were obtained from a slaughterhouse. The stage of estrous cycle (follicular or luteal) was identified. Follicular fluids (FF) were collected by puncture from three categories of follicles: small ( $\leq 5 \mathrm{~mm})$, medium $(6-10 \mathrm{~mm})$ or large $(>10 \mathrm{~mm})$ from ovaries in both follicular and luteal stage of estrous cycle.

The results indicate a significantly higher activity of SOD in bovine FF from follicles in luteal stage of estrous cycle than in follicular stage $(P=0.037)$. In FF of follicles in both stages of estrous cycle, SOD activity and BAP were significantly higher in FF of small and medium sized follicles than in large ones $(P=0.000$ both $)$. The ROMs significantly declined from small to large follicles in FF collected during both, luteal and follicular phase of estrous cycle $(P=0.005$ both). Activity of GPx showed no significant differences regarding neither estrous cycle nor size of follicles.

Results indicate similar antioxidative properties of bovine follicles during both luteal/follicular stage of estrous cycle with higher SOD activity in FF of follicles in luteal stage of estrous cycles which undergo atresia.

Keywords: BOVINE FOLLICULAR FLUID, ANTIOXIDATIVE SYSTEM, LUTEAL STAGE OF ESTROUS CYCLE, FOLLICULAR STAGE OF ESTROUS CYCLE

Follicular fluid (FF) accumulates in antral space and surrounds oocyte providing microenvironment for the development of oocytes. FF is a product of plasma filtration and of the secretory activity of granulosa and thecal cells. The molecular composition of follicular fluid includes proteins, nucleic acids and small molecules which show roles in cell nutrition and endocrine signaling and are likely to control the proliferation and development of granulose cells forming the cumulus around the oocyte [10].

Follicular fluid is responsible for providing oocyte protection against oxidation. The ovarian follicles contain macrophages, neutrophils and granulose cells producing reactive oxygen species (ROS) during metabolic processes. ROS must be continuously inactivated by nonenzymatic and enzymatic antioxidants to maintain balance between oxidant and antioxidants to preserve normal cell function [1]. The most important enzymatic molecules are superoxide dismutase (SOD) and glutathione peroxidase (GPx). Superoxide dismutase catalyses the dismutation of superoxide radical to hydrogen peroxide which will be removed by glutathione peroxidase. There were studies dealing with SOD function in oocyte development $[2,4,12,13]$. The reducing ability of nonenzymatic molecules (vitamin $\mathrm{C}$ and $\mathrm{E}$, glutathione and other protein thiols, bilirubin and uric acid) can be measured in biological samples by the ferric-reducing capacity [1].

The aim of the study was to examine activities of the superoxide dismutase, glutathione peroxidase and biological antioxidant potential 
(BAP) involved in protection against free oxygen radicals and concentration of reactive oxygen metabolites in bovine FF.

\section{Materials and methods}

\section{Follicular fluid collection and processing.}

The bovine ovaries $(n=76)$ were collected from naturally cycling animals at a local slaughterhouse and transported to the laboratory within $2 \mathrm{~h}$. Ovaries were transported in $0.9 \% \mathrm{NaCl}$ with antibiotics (100 I.U. penicillin/mL and $100 \mu \mathrm{g}$ streptomycin $/ \mathrm{mL}$ ) and stored at $4-8{ }^{\circ} \mathrm{C}$. In the laboratory, the stage of estrous cycle (follicular or luteal) was identified according to presence or absence of the corpus luteum on the ovary as previously described [6]. Follicular diameter was measured and follicles were classified according to diameter: small $(\leq 5 \mathrm{~mm})$, medium $(6-10 \mathrm{~mm})$ or large $(>10 \mathrm{~mm})$ follicles from ovaries in both follicular and luteal stage of estrous cycle. Ovaries with cystic follicles were excluded from the study. The FF was aspirated from each pair of ovaries using a $2 \mathrm{~mL}$ syringe attached to an 18-gauge needle. Follicular fluids from each group from each pair of the ovaries were pooled in one sample for each individual animal. The FFs were centrifuged ( $1600 \mathrm{~g}$ for $10 \mathrm{~min}$ ) for separation of the fluid from the cell fraction. Supernatants were stored at $-80^{\circ} \mathrm{C}$ until analysis.

Biochemical analyses. Activities of the superoxide dismutase (SOD), glutathione peroxidase (GPx) and biological antioxidant potential (BAP) involved in protection against free oxygen radicals and concentration of reactive oxygen metabolites (ROMs) were analysed in follicular fluids punctured from ovaries in follicular and luteal phase of estrous cycle. Glutathione peroxidase and SOD activities were assayed on an Olympus AU 400 (Olympus, Japan) biochemical analyzer, using the commercial Ransel and Ransod reagent kits (both Randox, Crumlin, UK), respectively. Concentrations of (ROM) and BAP were measured on an Olympus AU 400 (Olympus, Japan) biochemical analyser, using the commercial d-ROM and BAP reagent kits (both Diacron International, Italy), respectively.

Statistical analysis. All statistical analysis was performed using Statistica version 12 (Stat-
Soft, Palo Alto, California, USA). Data were expressed as means \pm standard deviation (SD). Data were analysed using way repeated measures ANOVA. Significance of follicles size differences within time was tested by Two-way mixed-design ANOVA followed by Tukey's significant difference test in case of normal distribution while a non-normal distribution by Kruskal-Wallis ANOVA and Dunn test for all groups. The level of $\mathrm{P}<0.05$ was considered statistically significant.

\section{Results and discussion}

Successful folliculogenesis may depend on balance between ROS and antioxidants. In this study we measured SOD and GPX activities and concentrations of BAP and ROM in bovine follicular fluid regarding to stage of estrous cycle (follicular and luteal) and follicular size.

SOD activity in follicular fluid originates from cells surrounding oocytes. SOD may play role in inhibition of progesterone synthesis [11], inhibition of aromatase activity [7] prevention of damaging effects of ROS and ovulation process [2]. In FF, SOD is responsible for reducing DNA damage and increasing oocyte cytoplasmic maturation [12]. We observed higher SOD activity in $\mathrm{FF}$ obtained from follicles during luteal phase ( $\mathrm{P}=0.037$; fig.). The similar results were obtained by [3]. In FF of follicles in both stages of estrous cycle, SOD activity was significantly higher in FF of small and medium sized follicles than in large ones $(\mathrm{P}=0.000$; table). Our results

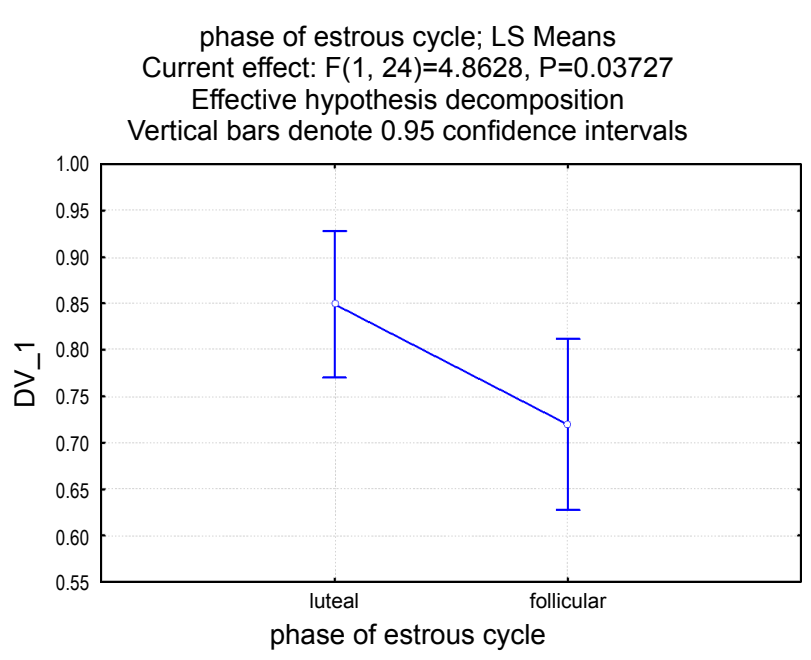

Fig. Bovine follicular fluid superoxide dismutase activity from follicles in luteal and follicular phase of estrous cycle 
Mean and standard deviation of activities of superoxide dismutase (SOD) and glutathione peroxidase (GPx) and concentrations of biological antioxidant potential (BAP) and reactive oxygen metabolites (ROM) in bovine follicular fluid from follicles in follicular and luteal phase of estrous cycle

\begin{tabular}{|l|c|c|c|c|c|c|}
\hline & \multicolumn{2}{|c|}{ small $(\leq 5 \mathrm{~mm})$} & \multicolumn{2}{c|}{ medium $(6-10 \mathrm{~mm})$} & \multicolumn{2}{c|}{ large $(>10 \mathrm{~mm})$} \\
\hline & follicular & luteal & follicular & luteal & follicular & luteal \\
\hline SOD (U/L) & $944 \pm 260 \mathrm{~A}$ & $963 \pm 229 \mathrm{a}$ & $694 \pm 264 \mathrm{AB}$ & $801 \pm 260 \mathrm{a}$ & $531 \pm 297 \mathrm{C}$ & $590 \pm 217 \mathrm{bc}$ \\
\hline GPx (U/L) & $265 \pm 107 \mathrm{~A}$ & $235 \pm 82 \mathrm{a}$ & $265 \pm 93 \mathrm{~A}$ & $291 \pm 69 \mathrm{a}$ & $274 \pm 88 \mathrm{~A}$ & $296 \pm 59 \mathrm{a}$ \\
\hline BAP (mm/L) & $3.63 \pm 0.21 \mathrm{~A}$ & $3.55 \pm 0.26 \mathrm{a}$ & $3.45 \pm 0.19 \mathrm{AB}$ & $3.31 \pm 0.35 \mathrm{a}$ & $3.06 \pm 0.30 \mathrm{C}$ & $3.26 \pm 0.20 \mathrm{bc}$ \\
\hline ROM (U CARR) & $106.43 \pm 37.41 \mathrm{AB}$ & $117.53 \pm 55.47 \mathrm{a}$ & $98.94 \pm 43.93 \mathrm{ABC}$ & $85.99 \pm 41.98 \mathrm{c}$ & $72.57 \pm 16.43 \mathrm{C}$ & $82.37 \pm 21.54 \mathrm{bc}$ \\
\hline
\end{tabular}

Note: Means, within same row, sharing the same superscript are not significantly different from each other $(\mathrm{P}<0.05)$. Uppercase and lowercase letters correspond to follicular and luteal phase of estrous cycle, respectively.

are in agreement with [1] in swine and [4] in buffalo FF. For ovulation certain concentration of ROS is needed [8] and maybe high SOD activity in FF prevent ovulation of follicles obtained during luteal phase.

The SOD contributes to the first line of antioxidant protection reducing damage converting superoxide radical to $\mathrm{H}_{2} \mathrm{O}_{2}$. If SOD activity is reduced, catalase activity is also decreased and GPx activity is increased [13]. In our research GPx shows a trend of increased activity during folliculogenesis $(\mathrm{P}=0.083$, table) which may indicate an increase in production of lipid peroxide in follicles during development. [1] reported significant decrease of GPx activity in follicular fluid from medium and large compared with small follicles.

The BAP test measures the concentration of different small nonenzymatic molecules having antioxidant properties such as bilirubin, uric acid vitamin $\mathrm{C}$ and $\mathrm{E}$ (Diacron International, Italy). In our research, BAP concentration was significantly higher in FF of small and medium sized follicles than in large ones regardless stage of estrous cycle $(\mathrm{P}=0.000)$. Our results are in agreement with [1] in swine FF. Results indicate that concentration of mentioned molecules decreased during bovine folliculogenesis and antioxidative protection depend on other compounds [5]. Positive correlation between ROS levels in FF and maturation parameters was found in different studies [9]. Among others, superoxide radicals, hydroxyl radical and $\mathrm{H}_{2} \mathrm{O}_{2}$ are the most important. d-ROM test measures hydroperoxides $(\mathrm{ROOH}$ which are products of superoxide and hydroxyl radicals peroxidation (Diacron International, Italy). In the present study, we showed that concentrations of ROMs during folliculogenesis are reduced in FF collected during both luteal and follicular phase of estrous cycle ( $\mathrm{P}=0.005$ both). [1] reported similar results on swine FF with the same test.

\section{Conclusions}

In conclusion, the major finding of the present study is higher SOD activity in FF obtained from follicles during luteal phase and decrease of ROMs level during follicle development. Higher level of SOD indicate lower concentrations of ROS species which are needed for ovulation and one of reasons for anovulation of follicles obtained during luteal phase maybe high SOD activity. Decreased concentration of ROMs suggests that oocyte during folliculigenesis is not subjected to oxidative stress and that balance between oxidants and antioxidants exists.

1. Basini G., Bussolati S., Santini S. E., Grasselli F. Reactive oxygen species and anti-oxidant defences in swine follicular fluids. Reproduction, Fertility and Development, 2008, vol. 20, issue 2, pp. 269-274. DOI: $10.1071 / \mathrm{RD} 07147$.

2. Combelles C. M. H., Holick E. A., Paolella L. J., Walker D. C., Wu Q. Profiling of SOD isoenzymes in compartments of the developing bovine antral follicle. Reproduction, 2010, vol. 139, issue 5, pp. 871-881. DOI: 10.1530/REP-09-0390.

3. El-Shahat K. H., Kandil M. Antioxidant capacity of follicular fluid in relation to follicular size and stage of estrous cycle in buffaloes. Theriogenology, 2012, vol. 77, issue 8, pp. 1513-1518. DOI: 10.1016/j.theriogenology.2011.11.018.

4. Hozyen H. F., Hodallah H. A., Essawy G. E. S., Shalaby S. I. A. Seasonal changes in some oxidant and antioxidant parameters during folliculogenesis in Egyptian buffalo. Animal Reproduction Science, 2014, vol. 151, issue 3-4, pp. 131-136. DOI: 10.1016/j.anireprosci.2014.10.005. 
5. Gupta S., Choi A., Yu H. Y., Czerniak S. M., Holick E. A., Paolella L. J., Agarwal A., Combelles C. M. H. Fluctuations in total antioxidant capacity, catalase activity, and hydrogen peroxide levels of follicular fluid during bovine folliculogenesis. Reproduction, Fertility and Development, 2011, vol. 23, issue 5, pp. 673-680. DOI: $10.1071 /$ RD10270.

6. Ireland J. J, Murphee R. L., Coulson P. B. Accuracy of predicting stages of bovine estrous cycle by gross appearance of the corpus luteum. Journal of Dairy Science, 1980, vol. 63, issue 1, pp. 155-160. DOI: 10.3168/jds.S0022-0302(80)82901-8.

7. LaPolt P. S., Hong L. S. Inhibitory effects of superoxide dismutase and cyclic guanosine 3 ', 5' - monophosphate on estrogen production in cultured rat granulosa cells. Endocrinology, 1995, vol. 136, issue 12, pp. 5533-5539. DOI: 10.1210/endo.136.12.7588305.

8. Miyazaki T., Sueoka K., Dharmarajan A. M., Atlas S. J., Bulkley G. B., Wallach E. E. Effect of inhibition of oxygen free radical on ovulation and progesterone production by the in-vitro perfused rabbit ovary. Journal of Reprodution and Fertility, 1991, vol. 91, issue 1, pp. 207-212. DOI: 10.1530/jrf.0.0910207.

9. Revelli A., Piane L. D., Casano S., Molinari E., Massobrio M., Rinaudo P. Follicular fluid content and oocyte quality: from single biochemical markers to metabolomics. Reproductive Biology and Endocrinology, 2009, vol. 7, p. 40. DOI: 10.1186/1477-7827-7-40.

10. Roy S. K. Regulation of Ovarian Follicular Development: A Review of Microscopic Studies. Microscopy Research and Technique, 1994, vol. 27, issue 2, pp. 83-96. DOI: 10.1002/jemt.1070270203.

11. Sugino N., Takiguchi S., Kashida S., Takayama H., Yamagata Y., Nakamura Y., Kato H. Suppression of intracellular superoxide dismutase activity by antisense oligonucleotides causes inhibition of progesterone production by rat luteal cells. Biology of Reproduction, 1999, vol. 61, issue 4, pp. 1133-1138. DOI: 10.1095/ biolreprod61.4.1133.

12. Tatemoto H., Muto N., Sunagawa I., Shinjo A., Nakada T. Protection of porcine oocytes against cell damage caused by oxidative stress during in vitro maturation: role of superoxide dismutase activity in porcine follicular fluid. Biology of Reproduction, 2004, vol. 71, issue 4, pp. 1150-1157. DOI: 10.1095/ biolreprod.104.029264.

13. Whitaker B. D., Knight J. W. Mechanisms of oxidative stress in porcine oocytes and the role of antioxidant. Reproduction, Fertility and Development, 2008, vol. 20, issue 6, pp. 694-702. DOI: 10.1071/RD08037. 